\section{Vernier acuity as affected by target length and separation*}

\section{G. D. SULLIVAN, K. OATLEY, and N. S. SUTHERLAND \\ University of Sussex, Brighton, Sussex, England}

Vernier acuity was measured for vertical lines of different lengths; the threshold (about $4 \mathrm{sec}$ of arc) was almost as good for the shortest stimuli (1 min 20 sec squares) as for the longest (21 min $20 \mathrm{sec} \times 1 \mathrm{~min} 20 \mathrm{sec}$ rectangles) and did not change when two round dots were shown in positions corresponding to the squares. The threshold for the two dots measured in terms of minimum detectable lateral offset increased when the vertical separation between the dots increased, but, when replotted in terms of the angle of tilt between them with respect to vertical, the threshold improved with dot separation; moreover, at asymptote, the threshold was comparable to that obtained for detecting that an actual line was tilted out of vertical. Our data suggest that, in performing the vernier task, Ss do not extrapolate the edges of the vernier elements; instead, they judge the deviation of the inner ends of the stimuli from verticality. This hypothesis explains the effect of increasing separation between vernier elements and also accounts for other types of acuity, such as the detection of curvature.

The threshold for vernier acuity (about 2 sec of arc under optimal conditions; Berry, 1948) is much less than the diameter of a foveal cone (about 24 sec of arc; O'Brien, 1951; Polyak, 1957). In an attempt to reconcile the fineness of the threshold with the dimensions of the receptor mosaic, Anderson and Weymouth (1923) argued that the brain averages the local pattern both over time (as successive fixations give rise to different images) and over the length of the stimulus lines. In support of their proposal, they reported experiments showing that acuity decreased both for short durations of exposure and for short line lengths.

Two recent experiments using stabilized images have shown that eye movements and, hence, the averaging over time of stimulation falling successively on different parts of the retina, do not play a crucial role in determining the vernier acuity threshold (Fender \& Nye, 1962; Keesey, 1960).

The idea that the fineness of vernier and stereoscopic acuity depends on averaging the position of a line or edge over its length seems to have more to recommend it. Indeed, it has often been shown (e.g., Hecht \& Mintz, 1939) that the contrast threshold for the detection of a line is a function of its length. Evidence relating vernier thresholds to line lengths is, however, much less conclusive. Averill \& Weymouth (1925) found that acuity for the detection of a step, offset in a black-white edge, "increases steadily until the length of the image equals the diameter of the fovea," and Anderson and Weymouth (1923) reached a similar conclusion for

*This work was financed by a grant from the Medical Research Council. essentially complete when the lines
One possible means of using this information is to detect the angle of tilt of the inner ends of the vernier elements. The vernier judgment then becomes one of orientation rather than a comparison of the position of edges.

The present experiments first provide further data on the question of whether the length of the vernier elements affects acuity; secondly, they directly compare the threshold in a conventional acuity task with the threshold for detecting that dots at varying vertical separations are out of vertical alignment-this task cannot be performed by extrapolating a straight edge since no straight edge is present in the stimulus; thirdly, the threshold for detecting that a line is tilted away from vertical was measured. If the vernier task is performed as a judgment of tilt, all three thresholds, when plotted in terms of angle of tilt from vertical, might be expected to yield comparable values. (1920) had reported that, for vernier acuity, the improvement that occurred with increasing line length was subtended $12 \mathrm{~min}$, and, in some of his conditions, it was complete within $4 \mathrm{~min}$ of arc. French's conclusions were based on only five settings per point, and he presented idealized curves rather than actual data.

It may be that vernier acuity involves neither averaging over time nor averaging over the length of an edge and then extrapolating its position. As we shall show later, such averaging is not necessary since, in principle, there is information in the retinal image that will allow the relative positions of two lines to be encoded to a much greater accuracy than the diameter of a single cone.

\section{METHOD \\ Apparatus}

Accurate displacements of one line with respect to another were produced by rotating through a small angle a sheet of plate glass placed in front of one line, and thus displacing its image laterally by refraction. Figure 1 is a sketch of the apparatus. An image displacement of $1.0 \mathrm{sec}$ of arc resulted from approximately $16 \mathrm{~min}$ of rotation of the lower sheet of glass. With no pattern displayed, rotations of the glass up to $8 \mathrm{deg}$ could not be detected by the Ss. Fixed displacements of the stimulus were provided by the preset stops and were accurate and repeatable to within 0.05 sec of arc. The prism device

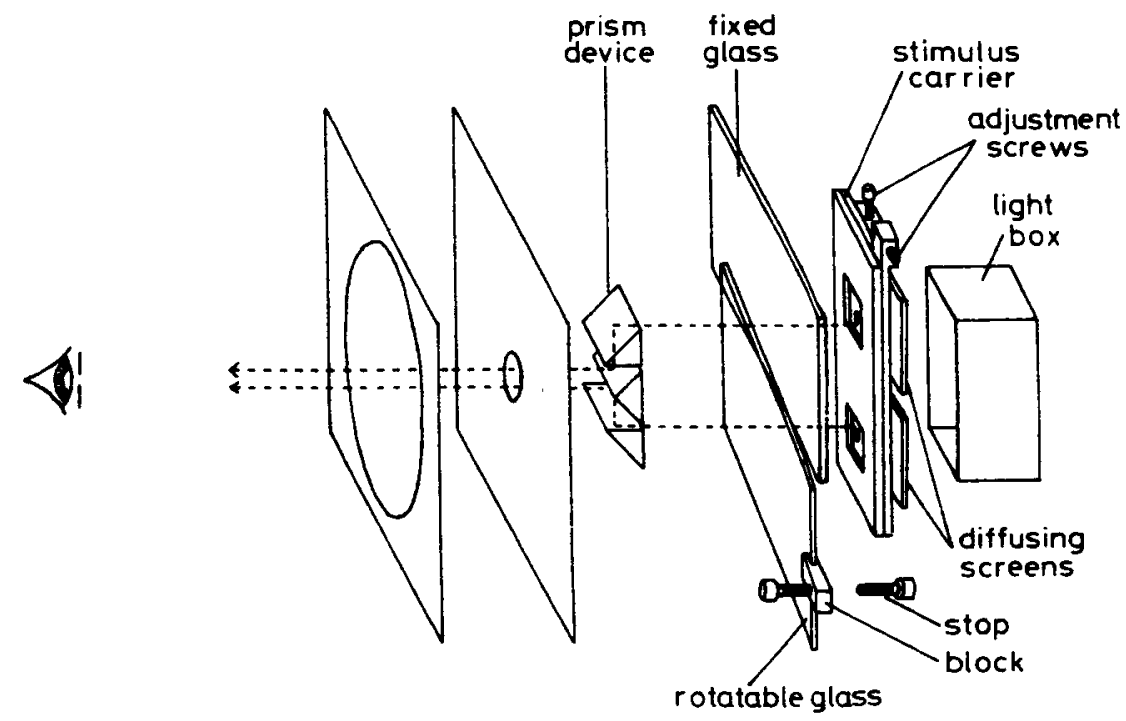

Fig. 1. Sketch of the apparatus. 

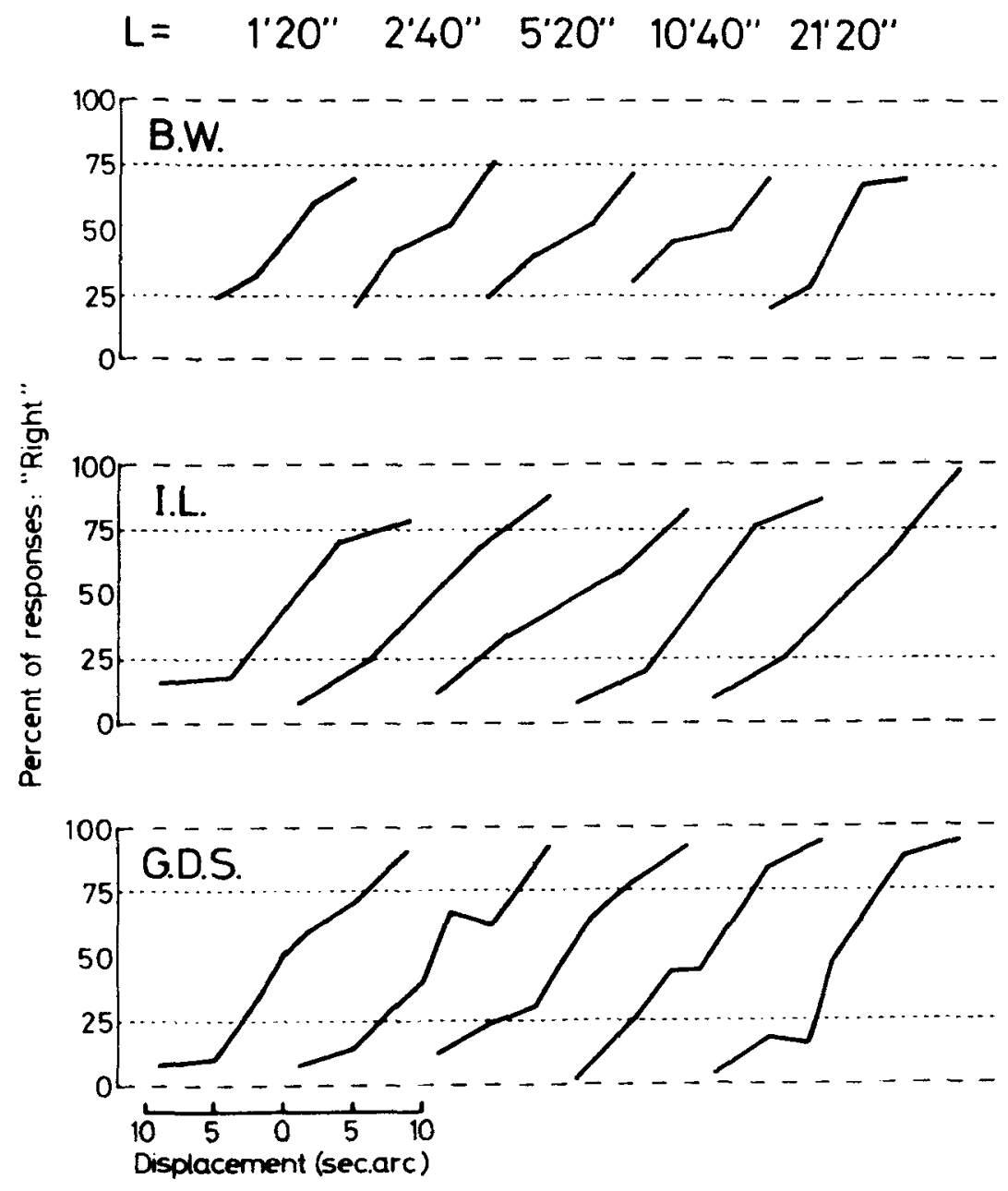

Fig. 2. Psychometric functions for stimuli without separating line.

eliminated the edges of the glass from the field of view. The apparatus was concealed by a white screen except for a circular field subtending $1.2 \mathrm{deg}$; the surround field subtended $8 \mathrm{deg}$. The center and the surround were matched to a luminance of $200 \mathrm{~cd} / \mathrm{m}^{2}$. The $\mathrm{S}$ held a fixed bite board between his teeth and viewed the display through a 2.3-mm artificial pupil, at a distance of $205 \mathrm{~cm}$.

\section{Stimuli}

The stimuli consisted of black lines or dots on transparent sheet film, transilluminated and viewed as shown in Fig. 1. They were mounted in carriers that slid into a spring-loaded channel, each carrier being equipped with adjustment screws so that they could be aligned for vertical position and orientation with the aid of a traveling telescope. We estimate that with the apparatus set to zero displacement, the lateral displacement of the vernier elements was within plus or minus 0.5 sec. Any inaccuracy did not, of course, affect the magnitudes of the displacements relative to one another for a particular stimulus.

\section{Subjects}

Three Ss were used, one of the a thors (G.D.S.) and two undergraduates (B.W. and I.L.) studying experimental psychology. Before the main experiments, all $\mathrm{Ss}$ had taken part in an extensive series of pilot experiments. They were, therefore, all thoroughly practiced in the vernier acuity task, and all had good acuity with uncorrected vision.

\section{General Procedure}

All the experiments were carried out using the method of constant stimuli with forced choices between two alternatives; after each trial, S signaled, by means of a switch, whether the bottom detail of the stimulus appeared to be to the left or to the right of the top one. Between trials, a rotary solenoid operated a shutter of lightly clouded Perspex, which obscured the stimuli and reduced the Iuminance by approximately $0.5 \mathrm{log}$ units. After a "Ready" signal from E, S triggered a timer that opened the shutter for 2.5 sec. The intertrial interval was between 4 and 6 sec.

Sessions of 35 or 40 trials were run at intervals of not less than $1 / 2 \mathrm{hr}$ during the experimental days. Before each session, Ss stayed for about $15 \mathrm{~min}$ reading in a constant environment in which the luminance of white paper was $12 \mathrm{~cd} / \mathrm{m}^{2}$. Each session was preceded by six warm-up trials, the results of which were not recorded. Within any one session, the same stimulus slide was used; the order of presentation of stimuli was counterbalanced across days. The sequence of displacements was random, except for two constraints. First, the same displacement was not used on consecutive trials; secondly, displacements to the same side did not occur on more than four consecutive trials. Within a session, each displacement was presented an equal number of times to left and right, and sufficient sessions were run to obtain 50 responses for each point on the psychometric function. The observed frequencies of response as a function of displacement were assumed to arise from normal-integral distributions. The best-fitting function was calculated by probit analysis (Finney, 1952), and its fit was evaluated by a chi-squared test; semiinterquartile displacements were taken as thresholds.

Before describing the experiments in detail, it is worth making two general comments on the results obtained. Out of 43 cases where an individual S's threshold was determined, the chi-squared value for the goodness of fit of the data to the integral normal curve was significant at better than the $5 \%$ level in only one instance and this result is to be expected by chance alone. Secondly, the form of the functions was very similar in all instances. Figure 2 shows an example of the psychometric functions obtained: the data are taken from the results obtained for each $S$ in Experiment 1, for vernier stimuli without a separating line.

\section{EXPERIMENT 1 \\ Procedure}

The stimuli were two vertical rectangles ( $1 \mathrm{~min} 20 \mathrm{sec}$ wide) separated by a 1 min $30 \mathrm{sec}$ gap. They were of two types. One type (see inset to Fig. 3) contained a blank gap between the vernier elements, and, in the other, a black horizontal separating line was present that exactly filled this gap (Fig. 4). The vertical extent of each rectangle (" $L$ " in the figures) was varied from $1 \mathrm{~min}$ $20 \mathrm{sec}$ (when each rectangle was 
square) up to $21 \mathrm{~min} 20 \mathrm{sec}$ in five steps, spaced at geometrical intervals.

The two undergraduates each received five daily sessions of 40 trials on 10 days spaced over 2 weeks. Two displacements to each side were presented, with values of 2 and 5 sec for B.W. and 4 and 9 sec for I.L. These values were chosen on the basis of previous measurements to produce points suitably placed on each S's psychometric function. G.D.S. was tested only with the stimuli without a separating line in this experiment, and psychometric function was obtained with zero displacement, together with 2, 5, and 9 sec on either side. For him, each displacement.

\section{Results}

For all Ss, the threshold hardly varied with line length for stimuli without the separating line (see Fig. 3). This flat function confirmed some preliminary observations on G.D.S. (not reported here in detail), using somewhat different apparatus in which the crack between the two mask. The mask was clearly visible, however, and we thought at the time that it provided a horizontal reference that enabled $S$ to judge when the two squares were not directly above one prism device to eliminate this clue. The results of the present experiment a more complete plot of the each session contained 35 trials, 5 with sheets of glass was obscured by a white another, and, hence, we inserted the

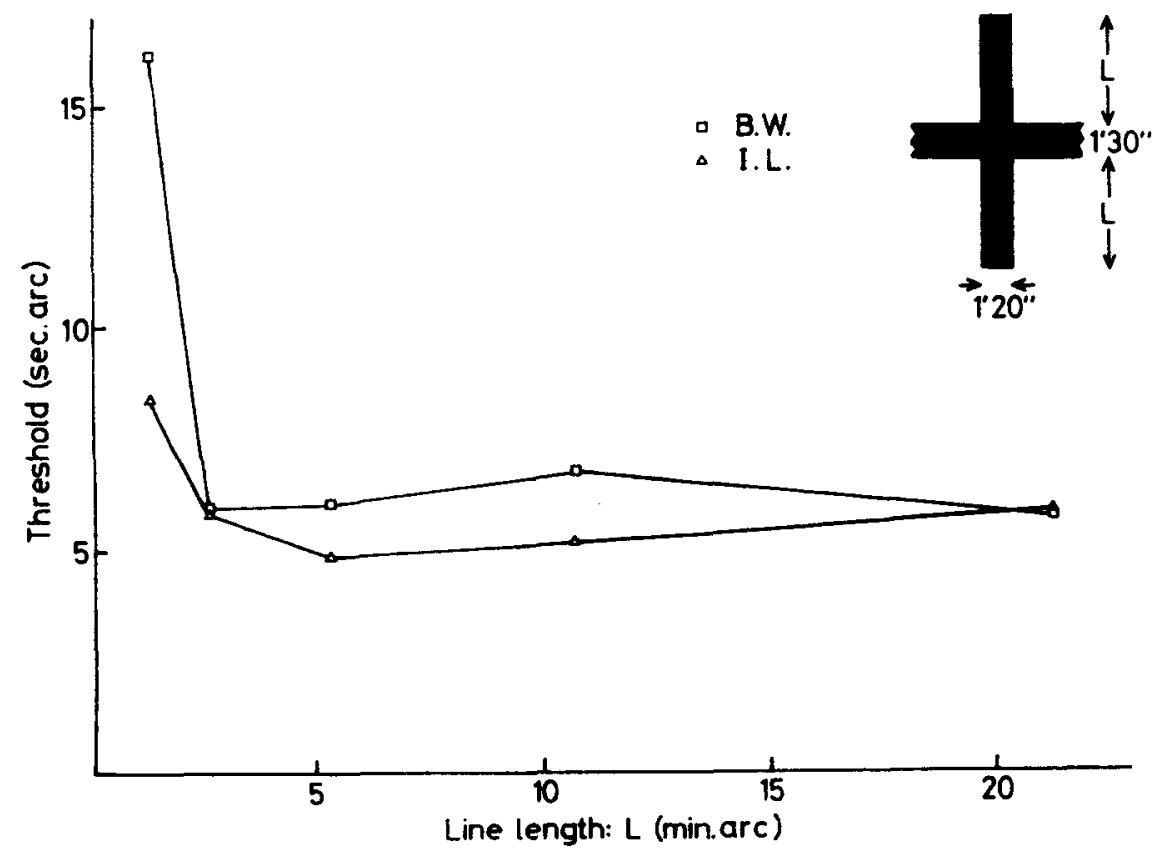

Fig. 4. Displacement thresholds for stimuli with separating line as a function of L. Data from Experiment 1.

show that, even without a visual reference orientation, the vernier task was performed as well with the two squares as with vertical lines. The mean threshold for the smallest stimuli used (1 min 20 sec squares) was $5.17 \mathrm{sec}$, which was little different from the threshold of $\mathbf{3 . 9 4} \mathrm{sec}$ for the

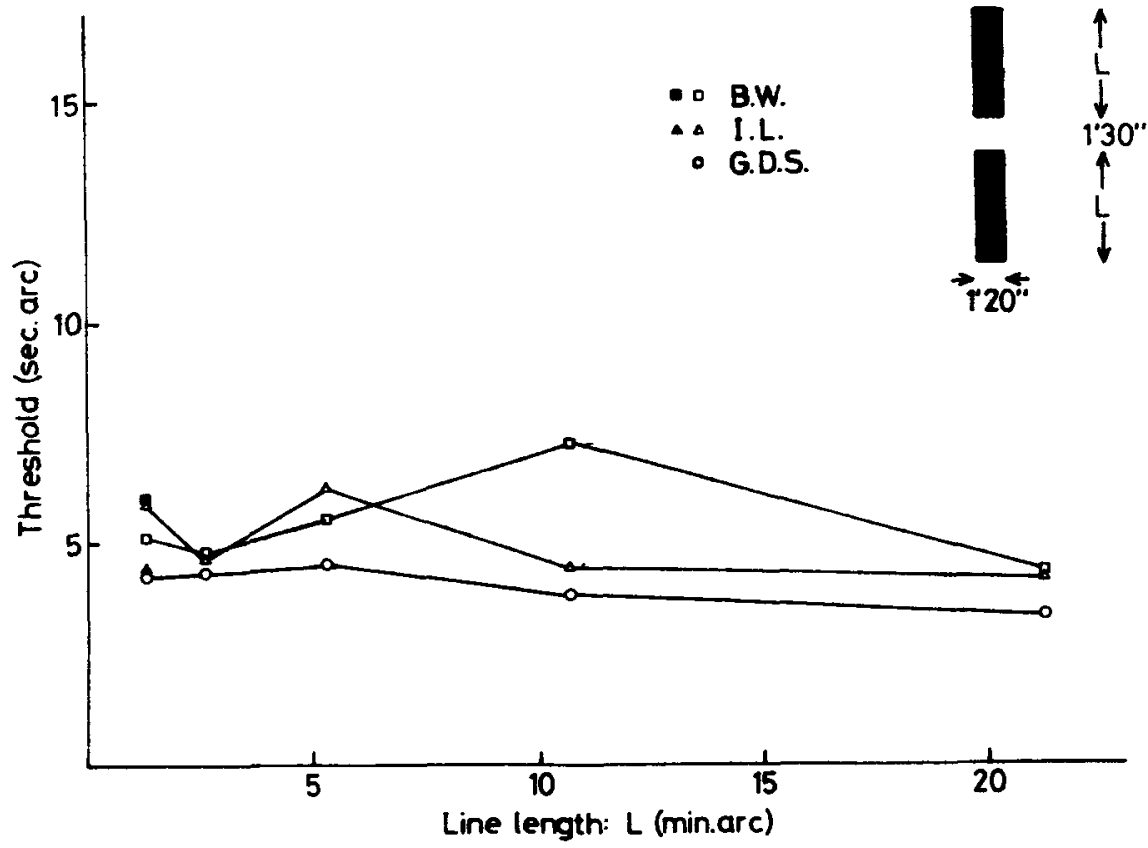

Fig. 3. Displacement thresholds for stimuli without separating line as a function of $\mathrm{L}$. Open symbols show data from Experiment 1 ; filled symbols show repeated measurement from Experiment 2. longest $(21 \mathrm{~min} 20 \mathrm{sec}$ ) lines.

Figure 4 displays the results of the two Ss for the stimuli with a black separating line. We had expected that reference enabling them to perform the task with the small squares better than in the condition where no horizontal line was present. However, for both $S s$, the reverse was the case, and, although the results displayed in Fig. 4 for the longer lines are very similar to those shown in Fig. 3, for the smallest stimuli, there was a sharp rise in threshold. This deterioration in acuity was accompanied by reports that the vernier elements in this condition were hardly visible, and B.W. said that on some trials she did not see them at all

When Ss gave their introspections about how they performed the task, they all said that they responded according to the configuration of the inner parts of the longer stimuli and ignored the rest of the rectangles. Curiously, when asked to draw the appearance of the stimuli, they drew displacements far larger than any that were, in fact, used. The two lines rarely seemed to be aligned, and apparent displacements of up to one-half the width of the rectangles (40 sec) were common.

\section{EXPERIMENT 2}

The results of the previous experiment strongly suggest that the task was not performed by extrapolation along the edges of the vernier elements. In order to confirm this horizontal line would provide a 


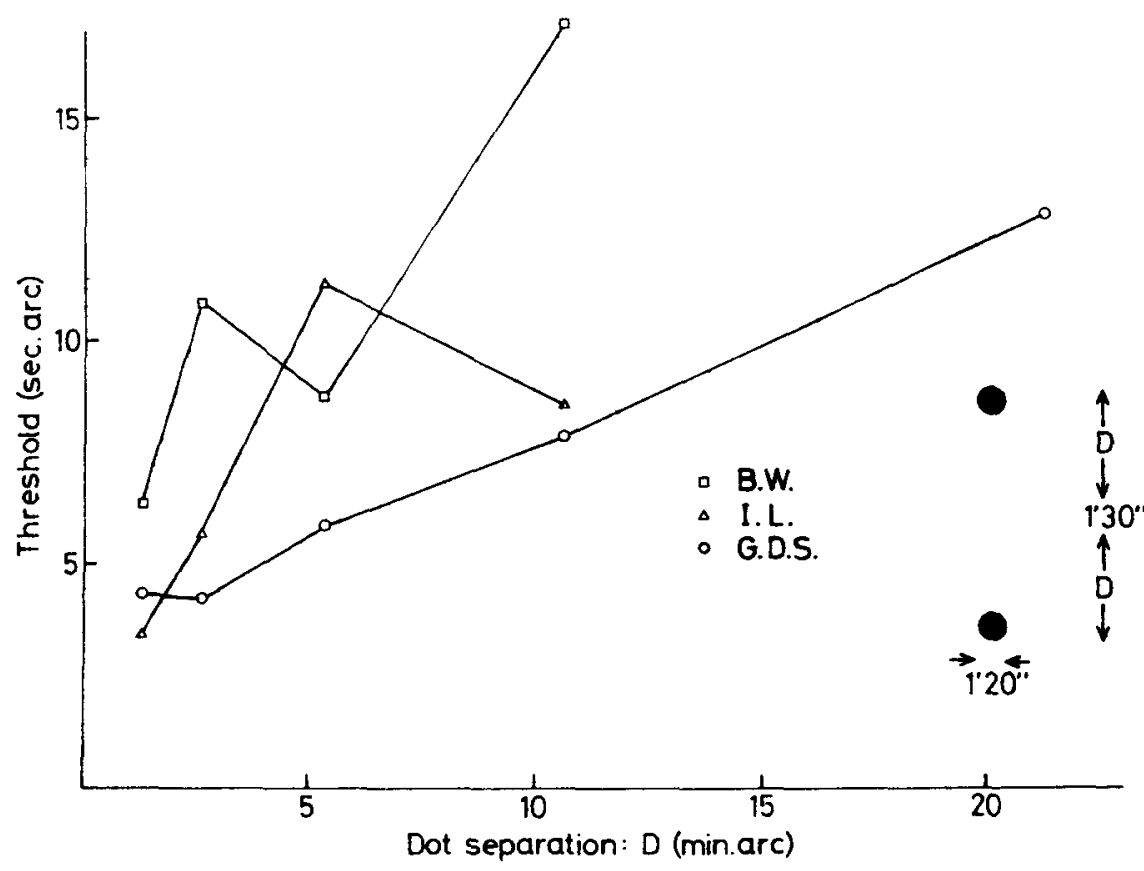

Fig. 5. Displacement thresholds for two-dot stimuli as a function of D. Data from Experiment 2.

this, we next tested the Ss with stimuli lacking vertical edges-namely, two round dots at varying separations.

\section{Procedure}

The dots were $1 \mathrm{~min} 20 \mathrm{sec}$ in diam; their separations were such that the positions of the dots corresponded to the outer ends of the line stimuli of Experiment 1. We refer to the separation by the measure $D$, which is equivalent to the measure $L$ of the line stimuli (compare the insets of Figs. 3 and 5). The two undergraduates were tested with four values of $D$ ranging from $1 \mathrm{~min} 20 \mathrm{sec}$ to $10 \mathrm{~min} 40 \mathrm{sec}$, and, in addition, we again determined acuity for the two squares without a separating line. These Ss had five sessions a day for 5 consecutive days, with displacements of 3 and $7 \mathrm{sec}$ on either side. G.D.S. was tested with five dot stimuli (D ranging from $1 \mathrm{~min}$ $20 \mathrm{sec}$ to $21 \mathrm{~min} 20 \mathrm{sec}$ ). For this $\mathrm{S}$, Experiments 1 and 2 were run simultaneously in a balanced design; the other procedural details were as given above.

\section{Results}

The functions of acuity against separation of the round dots for all three Ss are plotted in Fig. 5; the redetermined thresholds for the small squares for B.W. and I.L. are plotted in Fig. 3, and comparison with Fig. 5 shows that they lie close to those for dots. The functions displayed in Fig. 5 show an increase in threshold with increasing separation of the dots, as would be predicted if Ss were judging displacement by determining whether the line defined by the dots was displaced from vertical. Particularly for the more widely separated dots, the data for B.W. and I.L. are less reliable than those for G.D.S., reflecting the fact that, at greater separations, the larger displacements used for G.D.S. gave better estimates of the psychometric functions.

Incidentally, these results show that the failure to obtain an effect of line length in Experiment 1 was not due to the presence of some unsuspected clue in the apparatus, since, when widely separated dots were used, the threshold increased for all Ss.

\section{EXPERIMENT 3}

The foregoing results suggest that vernier acuity depends upon a judgment of orientation, and, hence, it seemed desirable to measure the Ss' thresholds for detecting the tilt of a single line from the vertical.

\section{Procedure}

The vernier displacement apparatus and the prism device were replaced by a rotary table from a milling machine. This held a piece of photographic film on which was a line subtending $23 \mathrm{~min}$ $12 \mathrm{sec} \times 1 \mathrm{~min} 27 \mathrm{sec}$ at a viewing distance of $188.5 \mathrm{~cm}$. (The line was designed to be seen at the viewing distance used in the other experiments $-205 \mathrm{~cm}$-but there was no space to insert the milling table at this distance. The $8 \%$ difference in viewing distance and line width between the present experiment and the others is unlikely to have had any effect on the results.) The milling table was calibrated in minutes of rotation and the drive had 3-min backlash, but, to increase accuracy, settings were made by winding from one direction. The Ss' task was to report whether the bottom of the single line was to the left or right of the top and was, therefore, comparable with the vernier tasks.

The undergraduates did five sessions of 40 trials on one experimental day, with essentially the same procedure as that used in the vernier experiments. Rotations of 10 and $25 \mathrm{~min}$ from the vertical to either side were presented. G.D.S. followed a routine similar to his previous one, with the line vertical and with rotations of 10,20 , and $30 \mathrm{~min}$ on both sides. Other details were as described above.

\section{Results}

The thresholds for perceived deviation from vertical are plotted in Fig. 6 as filled symbols. The results of the experiment with dots are also replotted on this figure in terms of a comparable angle of tilt from the vertical (open symbols). All Ss had a threshold of approximately $0.3 \mathrm{deg}$ for the tilt task, and, for two $\mathrm{Ss}$, this corresponded with the asymptotic level for the dot task. The surprising accuracy with which these judgments were performed without visual referents is probably partly accounted for by the stabilizing effect of the bite board and the fact that equal numbers of left and right trials of each displacement or tilt were given. This presumably helped to maintain an accurate mean adaptation level for subjective verticality.

\section{DISCUSSION}

The Nature of the Task

In addition to the Ss' introspections, the results suggest that the vernier task was performed by detecting that the direction defined by the inner edges of the vernier stimuli was tilted out of vertical. This hypothesis explains why acuity did not improve with increases in the length of the vernier elements, and why the threshold obtained with two dots was as good with two small squares; since the dots have no vertical edges, the former judgment could only have been made by detecting the angle of tilt between them. In fact, stimuli of this size (subtending only $1 \mathrm{~min}$ $20 \mathrm{sec}$ ) are below the threshold for form vision (cf. Casperson, 1950), and Ss reported that they saw them neither as squares nor as circles but as two blobs that sometimes fused together. In Experiment 2, where both types of stimuli were used, Ss could not tell them apart. 
Three further aspects of our results can best be understood on the assumption that judgments were made in terms of tilt.

(1) From geometrical considerations, the threshold should increase as the dot stimuli are separated by an increasing amount, since, as their vertical separation increases, larger lateral displacements are needed to produce a given angle of tilt between them. Fig. 5 shows this expected increase in the threshold measured in terms of lateral displacement.

(2) The judgment of offset cannot be better than the accuracy with which the $S$ can preserve a standard for the vertical. It follows that, if the vernier thresholds are expressed in terms of the angle of tilt made by the inner ends of the stimuli, then they should never be better than the threshold for detecting the tilt of a single line. Fig. 6 shows the thresholds for the two-dot stimuli replotted as the angle made by the two dots with the vertical, together with the results of Experiment 3, and confirms this expectation.

(3) It might be expected that the threshold for the two dots, plotted in terms of tilt, will increase with decreasing vertical separation between the dots, since, with smaller separations, inaccuracies in the precise localization of the dots would have a relatively greater effect on the angle of tilt of a line joining them. With small separations, therefore, the threshold will be limited by the accuracy of localization of the center of a dot or the end of a line, whereas for large vertical separations, it will be limited by the accuracy of the subjective norm for vertical. We might therefore expect a nonlinear curve, with threshold decreasing as a function of separation, and reaching asymptote at a value close to the threshold for judged line tilt. This expectation was again confirmed (see Fig. 6).

Our results are in good agreement with those of French (1920), who found little or no improvement in vernier acuity as the vernier elements were increased in length beyond $4 \mathrm{~min}$ of arc. Our data differ from those of Averill and Weymouth (1925), who found improvement as the vernier elements increased up to $30 \mathrm{~min}$ in length. There are several differences between their procedure and ours, but the discrepancy is probably accounted for by the fact that, instead of line elements with a vertical separation between them, they used a single edge containing a shoulder; i.e., either the top or the bottom half of the edge jutted out laterally beyond the remainder. The thresholds they

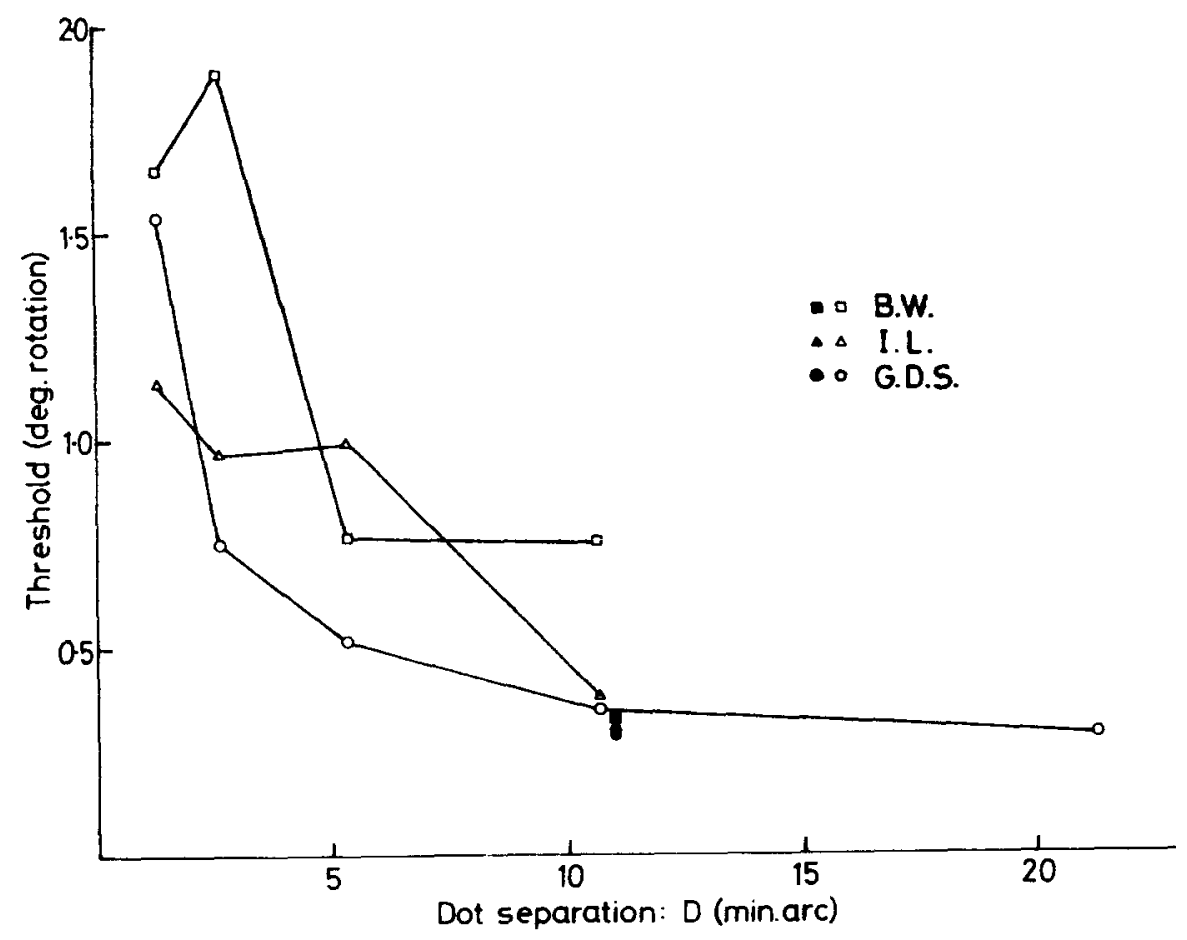

Fig. 6. Thresholds for two dot stimuli expressed as angle of rotation away from the vertical (open symbols), together with the thresholds for tilt of a single line from Experiment 3 (filled symbols).

obtained, even for the longest edges, were unusually high (on the order of $15 \mathrm{sec}$ of arc), and they report that "the offset was never seen as an abrupt angular break in the outline but one segment was slurred into the other in an inconspicuous manner and the judgment rested upon the fact that one segment failed to line up with the other." Averill and Weymouth's task is quite different from a conventional vernier task such as we used in Experiment 1. With their task, it would be difficult or impossible to use the tilt strategy employed by our own Ss, since their stimuli do not contain two clearly defined points between which it is possible to estimate an angle of tilt. Moreover, as explained above, as vertical separation becomes small, the accuracy with which orientation can be judged will depend critically on the accuracy with which the elements can be localized. Their Ss, therefore, must have used a different strategy, which resulted in thresholds that were much higher than those obtained by us and which were sensitive to edge length.

A similar difficulty with localizing clearly defined points may also explain the deterioration in acuity for the smallest vernier elements separated by a black horizontal line (Fig. 4).

Varieties of Tilt Judgment

If vernier tasks are performed by making a judgment of tilt, we would predict that, if the separation between conventional vernier lines were varied, the thresholds should not differ from the results of our Experiment 2 with dots. Berry (1948) performed such an experiment, varying the separation between two long (40-min) lines. He found that, for separations greater than $2 \mathrm{~min}$, the displacement thresholds were comparable to ours, but for smaller separations the threshold function dropped sharply to a well-defined minimum of $1.7 \mathrm{sec}$, at a separation of $20.1 \mathrm{sec}$. The smallest separation used in our expriments was $1.30 \mathrm{~min}$, so we have no evidence of any corresponding effect. We have recalculated Berry's data as tilt thresholds, and these are plotted in Fig. 7 (crosses) together with the results of G.D.S. from Fig. 6 (circles). When expressed in this way, the anomalous results for very small separations are still evident, but, with larger separations, there is good agreement between our results and Berry's. Thus, the tilt hypothesis accounts not only for the constancy of vernier thresholds as a function of line length, but also for their variation as a function of line separation.

French (1920) determined the threshold for setting a single line to vertical as a function of line length. We have also replotted his findings in Fig. 7 (dashed line), and his results are 


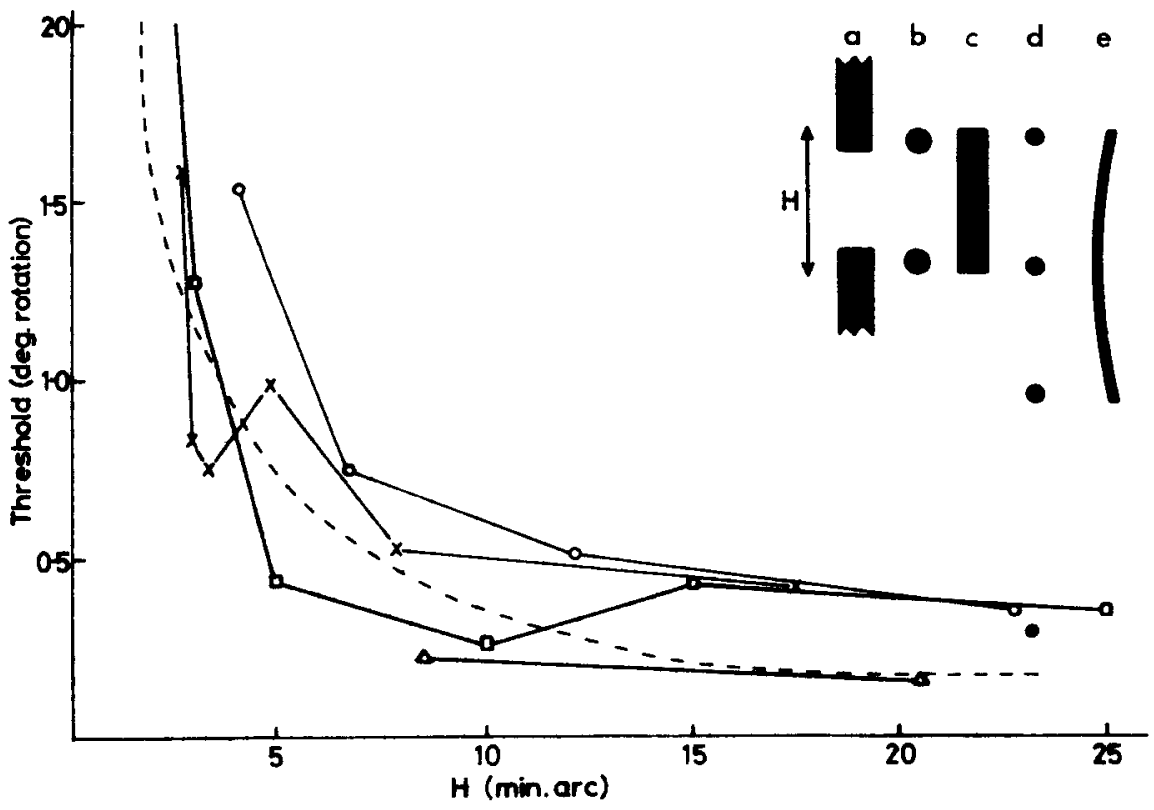

Fig. 7. Thresholds for five different acuity tasks expressed as angle of rotation about the vertical. As shown in the inset, vertical distance is in terms of our measure $H$. (a) Crosses: vernier threshold as a function of $H$, which is 2 min 40 sec longer than the separation of the vernier elements (from Berry, 1948). (b) Open circles: thresholds for two-dot stimuli as a function of separation (data of G.D.S. from Fig. 6). (c) Dashed line: threshold for tilt of a single line as a function of line length (from French, 1919); filled circle: similar task (G.D.S. from Fig. 6). (d) Squares: thresholds for the detection of the misalignment of three dots as a function of the separation of the dots (from Ludvigh, 1953). (e) Triangles: thresholds for the detection of curvature as a function of arc length (from Ogilvie \& Daicar, 1957 ).

again in good agreement with our own.

The tilt hypothesis encompasses two further types of acuity judgment. Ludvigh (1953) measured thresholds for detecting whether the center dot of three vertically arranged dots was aligned with the other two for vertical separations of 1.25 to 25 min between the center dot and each of the others; his results, replotted in terms of the threshold angle of tilt between the center and upper dot, are shown in Fig. 7 (squares), and they agree closely with those for the other tasks. Detection of curvature also fits into this scheme. Ogilvie and Daicar (1967) used two lengths of arcs of circles whose center points were tangential to the vertical and determined the threshold for curvature detection. Their results, recalculated as angles made by the chord from the center to the topmost point, are also plotted on Fig. 7 (triangles) and agree well with the others on that figure.

\section{Retinal Limitations}

In our task, the threshold for vernier stimuli, expressed as a lateral displacement, was only about one-fifth of a foveal cone diameter, and it did not deteriorate, even when the lines were reduced to a length subtending only about three foveal cones. These results raise again the fundamental problem of how such acuity is achieved. excitation of the cones by the two images.
Anderson and Weymouth (1923) believed that the all-or-none properties of nerve impulses implied all-or-none excitation of receptors and hence thought vernier acuity must involve averaging over time or over the length of the vernier elements. However, as Hartridge (1923) supposed then, and as is now universally recognized, receptors are capable of graded responses, and, therefore, details finer than the retinal grain can in principle be discriminated by an ensemble of receptors without such averaging. Figure 8 shows a row of cones and the (separate) retinal image distributions of two lines $1 \mathrm{~min} 20 \mathrm{sec}$ wide, laterally displaced with respect to one another by $10 \mathrm{sec}$, which represents the interquartile displacement when the threshold is 5 sec. Receptor dimensions are taken from Polyak (1957), and the image spread function from Byram (1944). The two rows of numbers in the cells represent the presumed amount of light exciting the receptors in the images of each line. To calculate these, we have integrated the light falling on each receptor and expressed this as a percentage of the excitation due to the bright background. The calculation has been carried out in one dimension only, but, as can be seen from Fig. 8 , there is a considerable difference in the excitation of the receptors by the two lines. It is difficult to argue a priori what is the minimum differential excitation that would lead to a just detectable difference in the receptor response, but there is no reason to
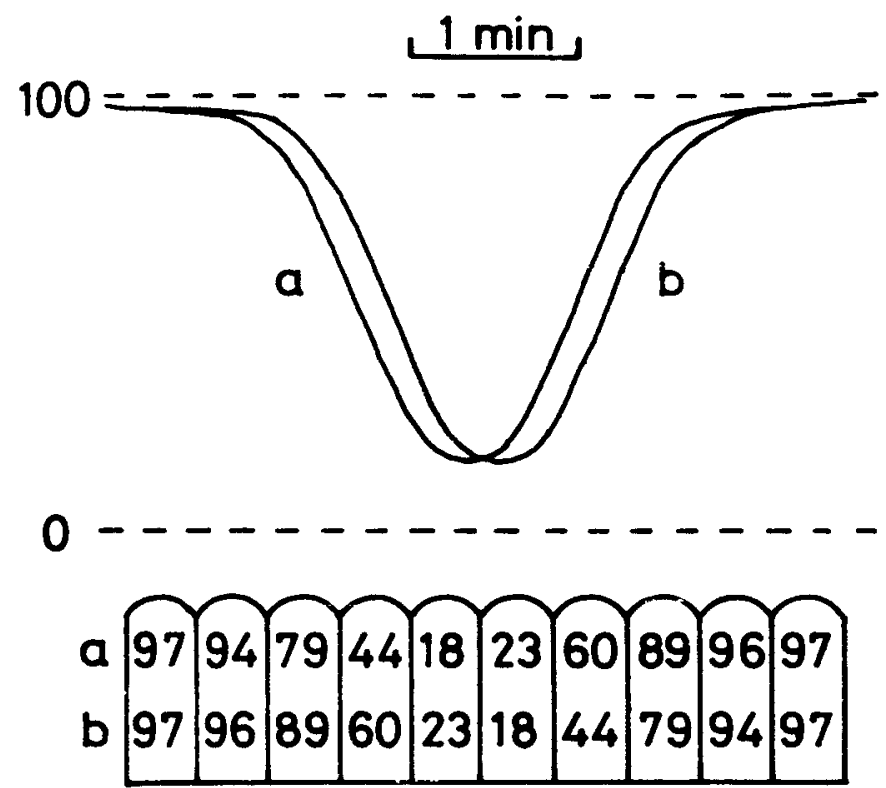

Fig. 8. Cross-sections of the (separate) retinal illuminance distributions of two black lines, 1 min 20 sec wide, displaced relative to one another by 10 sec. The cells represent a row of cones, and the two rows of numbers represent the 
suppose that the nervous system would not be sensitive to differences of the magnitude shown in Fig. 8 (i.e., differences of up to $30 \%$ ).

Neurophysiological Mechanisms

One question that can be raised is whether cortical units of the kind discovered by Hubel and Wiesel (1959, 1962) are implicated in vernier acuity. A possible role for such cells would be as detectors of collinearity. The response of a cell selective for the orientation of the vernier stimuli and having a receptive field overlapping both lines should diminish if the lines were offset. Such a mechanism would be sensitive to the length of the stimulus lines and, while some such process might account for Averill and Weymouth's (1925) results, it is clear that it cannot be applied to our findings.

When the vernier lines are reduced to two dots, the population of cortical cells excited by each of them would not be orientation specific; however, a pair of dots define an orientation, and cells tuned to this and neighboring orientations would be weakly excited. As the dot separation is decreased, cells responding to a wider range of orientations will be excited. A possible basis for the detection of the displacement of the dots would be to compute the mean orientation of the excited population of cells, and, as expected from this, the more specific cortical response to the widely separated dots is accompanied by better acuity when the thresholds are expressed as angle of tilt (Fig. 6). However, it is less easy to see how such a mechanism might be used in the conventional vernier task, since the weak response in the critical population of cells detecting the orientation of the ends of the lines might be expected to be masked by the strong response of cells responding selectively to the vertical bars. Paradoxically, we might expect the threshold to increase as the length of the vernier rectangles increases, but no such change in threshold occurred. However, little is know about the effects of combinations of stimuli on cells of the visual cortex. It is clear that, even if orientation sensitive cells are involved in vernier acuity, we are still some way from being able to give an account of the phenomena in these terms.

\section{REFERENCES}

ANDERSON, E. E., \& WEYMOUTH, F. W. Visual perception and the retinal mosaic. I. Retinal mean local sign-An explanation of the fineness of binocular perception of distance. American Journal of Physiology, 1923, 64, 561-594.

AVERILL, H. L., \& WEYMOUTH, F. W. Visual perception and the retinal mosaic. II. The influence of eye movements on the displacement threshold. Journal of Comparative Physiology; 1925, 5 , 147-176.

BERRY, R. N. Quantitative relations among vernier, real depth, and stereoscopic depth acuities. Journal of Experimental Psychology, 1948, 38, 708-721.

BYRAM G. M. The physical and photochemical basis of visual resolving power. Part I. The distribution of illumination in retinal images. Journal of the Optical Society of A merica, 1944, 34, 571-591.
CASPERSON, R. C. The visual discrimination of geometric forms. Journal of Experimental Psychology, $1950,40,668-681$.

FENDER, D. H., \& NYE, P. W. The effects of retinal image motion in a simple pattern recognition task. Kybernetik, $1962,1,192-199$.

FINNEY, D. J. Probit analysis. Cambridge: Cambridge University Press, 1952.

FRENCH, J. W. The unaided eye, Part III. Transactions of the Optical Society, 1919-1920, 21, $127-156$.

HARTRIDGE, H. Visual acuity and the resolving power of the eye. Journal of Physiology, 1923, 57, 52-67.

HECHT $S$ \& MINTZ, E. U. The visibility of single lines at various illuminations and the retinal basis of visual resolution. Journal of General Physiology, 1939, 22, 593-612.

HU BEL, D. H, \& WIFSEL, T. N. Receptive fields of single neurons in the cat's striate cortex. Journal of P hysiology, 1959, 148 , $574-591$.

HUBEL, D. H., \& WIESEL, T. N. Receptive fields, binocular interaction, and functional architecture in the cat's visual cortex. Journal of Physiology, 1962, 160, 106-154.

KEESEY, U. T. Effects of involuntary eye movements on visual acuity. Journal of the Optical Society of America, 1960, 50 769-774.

LUDVIGH, E. Direction sense of the eye. American Journal of Ophthalmology. $1953,36,139-143$.

$O$ 'BRIEN, B Vision and resolution in the central retina. Journal of the Optical Society of America, 1951, 41, 882-894. OGILVIE, J., \& DAICAR, E. The perception of curvature, Canadian Journal of Psychology, 1967, 21, 521-525.

POLYAK, S. L. The vertebrate visual system. Chicago: University of Chicago Press, 1957.

(Received for publication February 2, 1972; revision received June 30, 1972.) 